\title{
Posterior fossa pilocytic astrocytomas with oligodendroglial features show frequent FGFR1 activation via fusion or mutation
}

\author{
Philipp Sievers ${ }^{1,2}$, Daniel Schrimpf ${ }^{1,2}$, Damian Stichel ${ }^{1,2}$, David E. Reuss ${ }^{1,2}$, Martin \\ Hasselblatt $^{3}$, Christian Hagel ${ }^{4}$, Ori Staszewski ${ }^{5}$, Jürgen Hench ${ }^{6}$, Stephan Frank ${ }^{6}$, Sebastian \\ Brandner $^{7,8}$, Andrey Korshunov ${ }^{1,2,9}$, Wolfgang Wick ${ }^{10,11}$, Stefan M. Pfister ${ }^{9,12,13}$, Guido \\ Reifenberger ${ }^{14,15}$, Andreas von Deimling ${ }^{1,2}$, Felix Sahm ${ }^{1,2,9^{*}}$, David T. W. Jones ${ }^{9,16^{*}}$
}

1Department of Neuropathology, Institute of Pathology, University Hospital Heidelberg, Heidelberg, Germany

${ }^{2}$ Clinical Cooperation Unit Neuropathology, German Consortium for Translational Cancer Research (DKTK), German Cancer Research Center (DKFZ), Heidelberg, Germany

${ }^{3}$ Institute of Neuropathology, University Hospital Münster, Münster, Germany

${ }^{4}$ Institute of Neuropathology, University Medical Center Hamburg -Eppendorf, Hamburg, Germany

Institute of Neuropathology, Medical Faculty, University of Freiburg, Freiburg, Germany

${ }^{6}$ Institute for Medical Genetics and Pathology, University Hos pital Basel, Basel, Switzerland

${ }^{7}$ Division of Neuropathology, National Hospital for Neurology and Neurosurgery, University College London Hospitals NHS Foundation Trust, Queen Square, London, UK

${ }^{8}$ Department of Neurodegenerative Disease, UCL Queen Square Institute of Neurology, Queen Square, Lo nd on, UK

${ }^{9}$ Hopp Children's Cancer Center Heidelberg (KiTZ), Heidelberg, Germany

${ }^{10}$ Clinical Cooperation Unit Neurooncology, German Consortium for Translational Cancer Research (DKTK), German Cancer Research Center (DKFZ), Heidelberg, Germany

${ }^{11}$ Department of Neurology and Neurooncology Program, National Center for Tumor Diseases, Heidelberg University Hospital, Heidelberg, Germany

${ }^{12}$ Division of Pediatric Neurooncology, German Cancer Consortium (DKTK), German Cancer Research Center (DKFZ), Heidelberg, Germany

${ }^{13}$ Department of Pediatric Oncology, Hematology, Immunologyand Pulmonology, University Hospital Heidelberg, Heidelberg, Germany

${ }^{14}$ Ins titute of Neuropathology, Heinrich Heine University, Düsseldorf, Germany

${ }^{15}$ German Cancer Consortium (DKTK), Partner Site Essen/Düsseldorf, Germany

${ }^{16}$ Pediatric Glioma Research Group, German Cancer Research Center (DKFZ), Heidelberg, Germany

* These authors share senior authorship

Corresponding authors:

Felix Sahm, MD, PhD

Department of Neuropathology

Univ ersity Hospital Heidelberg

and

Clinical Cooperation Unit Neuropathology (B300)

German Cancer Research Center (DKFZ)

Im Neuenheimer Feld 224

69120 Heidelberg, Germany

Fon: +49-6221 56-37886

Fax: +49-6221564566

felix.sahm@med.uni-heidelberg.de

and

David T. W. Jones, PhD

Pediatric Glioma Research Group (B360)

Hopp Children's Cancer Center Heidelberg (KiTZ)

and

German Cancer Research Center (DKFZ)

Im Neuenheimer Feld 280

69120 Heidelberg, Germany

Fon: +49-6221 42-4675

Fax: +49-6221 42-4639

david.jones@dkfz.de

Keywords: Pilocytic astrocytoma; Brain tumor; Pediatric; Oligodendroglioma; DNA methylation profile; Molecular classification; FGFR1; FGFR1-TACC1 
Low-grade glial (LGG) and glioneuronal tumors (LGGNT) of the central nervous system (CNS) are an extremely diverse group of tumors with overlapping morphological features [6], making their histological diagnosis sometimes challenging. Molecular analyses can be very useful in distinguishing these entities from their clinical or histological mimics and raises the possibility of personalized targeted therapy [7,15]. In recent years, genome-wide DNA methylation profiling has proven to be a powerful technique to distinguish biological subgroups of brain tumors with characteristic alterations [1,3,11].

We investigated a challenging diagnostic case of a low-grade neuroepithelial tumor (LGNT) with focally discrete rosettes located in the mesencephalon by DNA methylation profiling, and subsequently identified a small group of neoplasms $(n=9)$ in a cohort of $>30,000$ tumors that were distinct from established DNA methylation profiles of other entities. Analyses and tissue acquisition are described in the supplementary material. Unsupervised hierarchical clustering and t-distributed stochastic neighbor embedding (t-SNE) analysis of DNA methylation patterns alongside other, well-characterized LGNT entities, confirmed the distinct nature of this class (Fig.1). Analysis of copy number profiles (CNPs) showed evidence for a fusion between fibroblast growth factor receptor 1 (FGFR1) and transforming acidic coiled-coil containing protein 1 (TACC1) genes (Fig.1) in four of nine of the tumors. A subsequent transcriptome and targeted next-generation sequencing analysis $[9,13]$ revealed alterations (fusions or mutations) within the FGFR1 signaling pathway in all cases with sufficient material $(n=8)$. Six of eight tumors $(75 \%)$ demonstrated an FGFR1-TACC1 fusion and two (25\%) a hotspot mutation within the kinase domain of FGFR1 resulting in a c.1638C>A, p.N546K or c.1966A>G, p.K656E substitution (Fig.1 and Supplementary Table 1). The mutant allele frequency for both FGFR1 mutations was $39 \%$, consistent with a heterozygous somatic mutation. Immunohistochemical detection of phospho-FGFR1 showed diffuse cytoplasmic positivity in all tumors harboring an FGFR1 alteration (Fig. 2). FGFR1 encodes the fibroblast growth factor receptor 1, a tyrosine-protein kinase that mainly acts via the MAPK and PIBK pathways. Genetic alterations within the FGFR signaling pathway are common in LGGs and LGGNTs, with missense mutations in FGFR1 and kinase domain duplications being the most frequent events $[4,7,8,15]$. In particular, this includes entities such as pilocytic astrocytoma [4], dysembryoplastic neuroepithelial tumor [8], rosette-forming glioneuronal tumor [10] and extraventricular neurocytoma (where fusions are more common) [11]. In addition, FGFR1 alterations are occasionally found in glioblastoma [12]. Aberrant activation of the FGFR1 pathway is also an attractive molecular target from a therapeutic perspective, since highly potent FGFR inhibitors are currently in clinical trials [5]. These novel treatment options could be of particular value in patients with subtotally resected or diffusely infiltrating tumors, with indication for further therapy. Interestingly, one of the FGFR1-mutant tumors also harbored two NF1 alterations, resulting in c.7006delG, p.A2336fs and c.C2293T, p.R765C. While the frameshift event is likely to be pathogenic, the biological relevance of the missense mutation remains uncertain. Germline material was not available from this patient. Although there are reports of concomitant mutations in LGGs and LGGNTs with potentially overlapping effects on the MAPK/ERK pathway, the vast majority of these tumors typically have only one pathway alteration [2,15]. Besides FGFR1 and NF1 alterations, no additional pathogenic mutations were detected (particularly no BRAF, IDH1/2, H3, ATRX, TP53, CIC, FUBP1 or TERT promoter mutations), and none of the tumors harbored a $1 \mathrm{p} / 19 \mathrm{q}$ codeletion (Supplementary Table 1).

Most of the tumors were located within the posterior fossa, with exceptions arising in the temporal lobe and in the mesencephalon. Median age at presentation was nine years (range 
$1-31 ; 8$ of 9 patients younger than 18 years) and the sex distribution was not significantly biased considering the small number of patients (male:female ratio 1.25:1; Fig. 1 and Supplementary Table 1). Treatment and outcome data were unfortunately not available for the majority of the cohort, and further studies will be needed to investigate any prognostic significance of this molecular constellation.

The original histological diagnosis for the majority of the tumors $(n=7)$ was pilocytic astrocytoma World Health Organization (WHO) grade I, with single cases diagnosed as LGG or LGGNT (Fig. 1). In light of their common molecular features, we also reviewed all cases histologically.

All tumors were characterized by a moderate to high cellularity of monomorphic or slightly pleomorphic neoplastic cells with round and partly hyperchromatic nuclei. Most $(n=8)$ harbored areas exhibiting an extensive or focal oligodendroglial morphology with perinuclear halos (Fig. 2 and Supplementary Table 2). Multinucleated cells with nuclei arranged in a 'pennies on a plate' configuration [6] were seen in only one of the tumors. In three cases, tumor cells were focally arranged in ribbons. A microcystic architecture was seen in all cases. Most of the tumors demonstrated either eosinophilic granular bodies $(n=6)$ or Rosenthal fibers $(n=3)$. Extensive calcification was seen in a small number of tumors $(n=3)$. Focal capillary proliferation was observed in five of the cases. Necrosis was uniformly absent. Mitotic activity was very low to absent. Ki-67 labeling activity was $1-2 \%$ in seven tumors, while two tumors had a focally elevated proliferation index of up to $5 \%$. All tumors exhibited immunohistochemical expression of glial fibrillary acidic protein (GFAP). Five tumors displayed a weak cytoplasmatic immunoreactivity for synaptophysin. MicrotubuleAssociated Protein 2 (MAP2) positivity was focally detected in six tumors. Expression of NeuN was entirely negative in all tumors. Altogether, the histological features of all tumors could be considered to be broadly consistent with current descriptions of the morphological spectrum of pilocytic astrocytoma $[6,14]$.

In summary, we have identified a molecularly distinct subset of low-grade glial tumors by DNA methylation profiling with recurrent alterations affecting FGFR1, which most commonly arises in the cerebellum and which is histologically enriched for the presence of oligodendroglial morphology in a pilocytic astrocytoma-like background. The prominent cerebellar location and initial diagnosis as pilocytic astrocytoma in most cases suggests that this could represent an FGFR-activated subset of (cerebellar) pilocytic astrocytoma, in contrast to the KIAA1549-BRAF fusion that is by far the most commonly observed alteration in this context. Furthermore, the prominent oligodendroglial component of these tumors suggests that they may account for a proportion of tumors still occasionally considered as 'pediatric-type oligodendroglioma'. These findings further support the utility of FGFR1 molecular testing as part of the diagnostic assessment of pediatric low-grade glial or glioneuronal tumors and identifies a tumor subset with a potential therapeutic target that may be of interest in a subset of patients with sub-totally resected, progressive and/or disseminated disease.

Acknowledgments For excellent technical support we sincerelythank the Microarray Unit of the German Cancer Research Center (DKFZ) Genomics and Proteomics Core Facility, as well as U. Vogel, A. Habel and S. Kocher (Department of Neuropathology, Institute of Pathology, University Hospital Heidelberg, Heidelberg, Germany). We are grateful to Kathy Keyvani for kindly providing tumor tissue (Institute of Neuropathology, University of Duisburg-Essen, Essen, Germany). D.T.W. Jones is supported by the Everest Centre for Low-grade Paediatric 
Brain Tumours (The Brain Tumour Charity, UK). F. Sahm is a fellow of the Else Kröner Excellence Program of the Else Kröner-Fresenius Stiftung (EKFS). S. Brandner is supported by the UK Department of Health's NIHR Biomedical Research Centre's funding scheme. 


\section{References}

1. Capper D, Jones DTW, Sill M, Hovestadt V, Schrimpf D, Sturm D et al. (2018) DNA methylationbased classification of central nervous system tumours. Nature 555:469-474. doi:10.1038/nature26000

2. D'Angelo F, Ceccarelli M, Tala, Garofano L, Zhang J, Frattini V et al. (2019) The molecular landscape of glioma in patients with Neurofibromatosis 1. Nat Med 25:176-187. doi:10.1038/s41591018-0263-8

3. Deng MY, Sill M, Chiang J, Schittenhelm J, Ebinger M, Schuhmann MU et al. (2018) Molecularly defined diffuse leptomeningeal glioneuronal tumor (DLGNT) comprises two subgroups with distinct clinical and genetic features. Acta Neuropathol. doi:10.1007/s00401-018-1865-4

4. Jones DT, Hutter B, Jager N, Korshunov A, Kool M, Warnatz HJ et al. (2013) Recurrent somatic alterations of FGFR1 and NTRK2 in pilocytic astrocytoma. Nat Genet 45:927-932. doi:10.1038/ng.2682

5. Lasorella A, Sanson M, lavarone A (2017) FGFR-TACC gene fusions in human glioma. Ne uro Oncol 19:475-483. doi:10.1093/neuonc/now240

6. Louis DN, Ohgaki H, Wiestler OD, Cavenee WK (2016) WHO Classification of Tumours of the Central Nervous System. Revised 4th edition edn. IARC, Lyon

7. Qaddoumi I, Orisme W, Wen J, Santiago T, Gupta K, Dalton JD et al. (2016) Genetic alterations in uncommon low-grade neuroepithelial tumors: BRAF, FGFR1, and MYB mutations occur at high frequency and align with morphology. Acta Neuropathol 131:833-845. doi:10.1007/s00401-016-1539-z

8. Rivera B, Gayden T, Carrot-Zhang J, Nadaf J, Boshari T, Faury D et al. (2016) Germline and somatic FGFR1 abnormalities in dysembryoplastic neuroepithelial tumors. Acta Neuropathol 131:847863. doi:10.1007/s00401-016-1549-x

9. Sahm F, Schrimpf D, Jones DT, Meyer J, Kratz A, Reuss D et al. (2016) Next-generation seque ncing in routine brain tumor diagnostics enables an integrated diagnosis and identifies acti onable targets. Acta Neuropathol 131:903-910. doi:10.1007/s00401-015-1519-8

10. Sievers P, Appay R, Schrimpf D, Stichel D, Reuss DE, Wefers AK et al. (2019) Rosette-forming glioneuronal tumors share a distinct DNA methylation profile and mutations in FGFR1, with recurrent co-mutation of PIK3CA and NF1. Acta Neuropathol. doi:10.1007/s00401-01902038-4

11. Sievers P, Stichel D, Schrimpf D, Sahm F, Koelsche C, Reuss DE et al. (2018) FGFR1:TACC1 fusion is a frequent event in molecularly defined extraventricular neurocytoma. Acta Neuropathol 136:293-302. doi:10.1007/s00401-018-1882-3

12. Singh D, Chan JM, Zoppoli P, Niola F, Sullivan R, Castano A et al. (2012) Transforming fusions of FGFR and TACC genes in human glioblastoma. Science 337:1231-1235. doi:10.1126/science.1220834

13. Stichel D, Schrimpf D, Casalini B, Meyer J, Wefers AK, Sievers P et al. (2019) Routine RNA sequencing of formalin-fixed paraffin-embedded specimens in neuropathology diagnostics identifies diagnostically and therapeutically relevant gene fusions. Acta Neuropathol. doi:10.1007/s00401-019-02039-3

14. Tihan T, Ersen A, Qaddoumi I, Sughayer MA, Tolunay S, Al-Hussaini M et al. (2012) Pathologic characteristics of pediatric intracranial pilocytic astrocytomas and their impact on outcome in 3 countries: a multi-institutional study. Am J Surg Pathol 36:43-55. doi:10.1097/PAS.0b013e3182329480

15. Zhang J, Wu G, Miller CP, Tatevossian RG, Dalton JD, Tang B et al. (2013) Whole-genome sequencing identifies genetic alterations in pediatriclow-grade gliomas. Nat Genet 45:602612. doi:10.1038/ng.2611 


\section{Figure legends}

Fig. 1 Molecular classification of posterior fossa pilocytic as trocytomas with oligodendroglial features (PA PF FGFR1) by DNA Methylation Profiling.

a) Unsupervised hierarchical clustering of DNA methylation profiles of nine PAPF FGFR1 alongside 130 wellcharacterized glial and glioneuronal reference samples and control tissue using the 20,000 most variably methylated probes. Reference methylation classes: posterior fossa pilocytic astrocytoma (LGG, PA PF), polymorphous low-grade neuroepithelial tumor of the young (PLNTY), ganglioglioma (LGG, GG), midline pilocytic as trocytoma (LGG, PA MID), supratentorial/hemispheric pilocytic as trocytoma and ganglioglioma (LGG, PA/GG), diffuse leptomeningeal glioneuronal tumor subgroup 1 (DLGNT_1), diffuse leptomeningeal glioneuronal tumor subgroup 2 (DLGNT_2), low-grade glioma with MYB or MYBL1 rearrangement (LGG_MYB), extraventricular neurocytoma (EVN), dysembryoplastic neuroepithelial tum or (DNT), rosette-forming glioneuronal tumor (RGNT), papillaryglioneuronal tumor (PGNT), central neurocytoma (CN) and control tis sue white matter (CONTROL).

b) Two-dimensional representation of pairwise sample correlations using the 15,000 most variant probes by tdistributed stochastic neighbor embedding (t-SNE) dimensionality reduction (same samples as in a).

c) Schematic fusion configuration of case 8 showing an FGFR1-TACC1 fusion.

d) Clinicopathological characteristics and key genetic alterations identified in the PA PF FGFR1 cohort. Abbreviations:PA, Pilocytic astrocytoma WHO grade I; GTA, Glial tumor with features of anaplasia; LGGNT, Lowgrade glioneuronal tumor.

Fig. 2 Morphological and immunohistochemical features of selected tumors from the PA PF FGFR1 cohort. Monomorphic cells with an oligodendroglial morphology (a), expression of FGFR1 in PA PF FGFR1 (b) and control tissue (c).

Supplementary Fig. 1 Copy-number profile derived from DNA methylation array of the FGFR1-mutant tumor harboring a concomitant mutation in NF1.

Supplementary Fig. 2 Morphological and immunohistochemical features seen in the PA PF FGFR1 cohort. Hematoxylin and eosin (HE)-stained sections show monomorphic cells with round uniform nuclei with perinuclear halos resembling oligodendroglioma $(a, b)$. A subset of tumors is focallyarranged in ribbons (c). Typical biphas ic pattern of pilocytic astrocytoma with a loose, myxoid component and a more compact component (d) showing Rosenthal fibres and eosinophilic granular bodies (e). Calcifications (f). Bipolar cells with long, narrow proces s es (g) and multinucleated cells with nuclei arranged in a 'pennies on a plate' configuration (h). Glomeruloid microvascular proliferations (i). Immunohistochemically, tumors are GFAP-positive (j) whereas MAP2 (k) and synaptophysin (I) expression is observed onlyin single cases. Scale bars $100 \mu \mathrm{m}$. 